\title{
Initial experience of pulmonary embolism response team with percutaneous embolectomy in intermediate-high- and high-risk acute pulmonary embolism
}

\author{
Marek Roik ${ }^{1}$, Dominik Wretowski ${ }^{1}$, Andrzej Łabyk ${ }^{1}$, Michał Ciurzyński ${ }^{1}$, Katarzyna Kurnicka ${ }^{1}$, \\ Barbara Lichodziejewska ${ }^{1}$, Szymon Pacho $^{1}$, Michał Potępa ${ }^{1}$, Agnieszka Szramowska ${ }^{1}$, \\ Janusz Trzebicki², Marek Gołębiowski ${ }^{3}$, Piotr Pruszczyk ${ }^{1}$ \\ 'Department of Internal Medicine and Cardiology, Medical University of Warsaw, Warsaw Poland \\ ${ }^{2} 1^{\text {st }}$ Department of Anaesthesiology and Intensive Care Unit, Medical University of Warsaw, Warsaw, Poland \\ ${ }^{3} 1^{\text {st }}$ Department of Radiology, Medical University of Warsaw, Warsaw, Poland
}

\section{INTRODUCTION}

Shock or persistent hypotension affects $5 \%$ of patients with pulmonary embolism (PE) and identifies a high-risk group with PE-related mortality exceeding $15 \%$ [1, 2]. Primary reperfusion, particularly systemic thrombolysis, is the treatment of choice. Due to high bleeding risk, active malignancies, or multiple medical comorbidities, only a minority of unstable patients undergo thrombolysis [2, 3]. Up till now, when this treatment was contraindicated or unsuccessful, a surgical embolectomy has been recommended. Unfortunately, due to its limited immediate availability, surgical embolectomy can only be performed in selected high-risk patients. Significant progress in interventional cardiology has made percutaneous catheter-directed thrombectomy (CDT) an alternative to surgical embolectomy [4-15]. CDT of PE may encompass mechanical or ultrasound-assisted clot fragmentation, aspiration, and local low-dose fibrinolytic injection [1, 7-10, 13]. CDT was proposed as an integrated part of the treatment offered by a pulmonary embolism response team (PERT) $[1,11,12]$. According to our data, due to high prevalence of absolute contraindications, only approximately $40 \%$ of haemodynamically unstable patients received thrombolysis therapy [3]. In May 2015, we established a local PERT with CDT capabilities for urgent invasive therapy of intermediate-high- or high-risk PE in patients with high bleeding risk or those after unsuccessful thrombolysis. We report our initial experience of a single-centre PERT in the treatment of intermediate-high- or high-risk PE with rheolytic percutaneous embolectomy.

\section{METHODS}

Our PERT consists of an interventional cardiologist, intensivist, radiologist, and a clinical cardiologist with experience in echocardiography and vascular ultrasound. The PERT is called to high-risk PE patients with absolute contraindications to thrombolysis or those after an unsuccessful primary/rescue thrombolysis, as well as to intermediate-high-risk PE patients who fail to respond to parenteral anticoagulation. CDT is performed in a cardiac catheterisation laboratory under unfractionated heparin administration (a bolus of $70 \mathrm{IU} / \mathrm{kg}$ followed by intravenous infusion adjusted to the activated partial thromboplastin time (aPTT) [1]. After exclusion of proximal lower limb venous thrombosis with bedside ultrasound, selective pulmonary angiography is performed with a $5 \mathrm{~F}$ or $6 \mathrm{~F}$ angled pigtail catheter via a femoral or jugular vein approach. An 8 F 55- or 90-cm guiding catheter is inserted into the pulmonary artery and an Angiojet percutaneous thrombectomy system (Medrad Inc., Warrendale, PA, USA) is advanced into the proximal pulmonary artery beyond the thrombus. Individual catheter activation times are limited to $20 \mathrm{~s}$, and the system is stopped if clinical bradycardia or dyspnoea occurs. The total Angiolet activation time is limited to 3 min for treatment of unilateral PE and 4 min for bilateral PE. Rheolytic thrombectomy is stopped either when haemodynamic stabilisation occurs or when the maximal activation time is reached. Following CDT all patients are transferred to the coronary care unit with continuous unfractionated heparin infusion adjusted to the aPTT [1].

Address for correspondence:

Marek Roik, MD, PhD, Department of Internal Medicine and Cardiology, ul. Lindleya 4, 02-005 Warszawa, Poland, tel: +48 225021144 , fax: +48 225021363 , e-mail:mroik@wum.edu.pl 


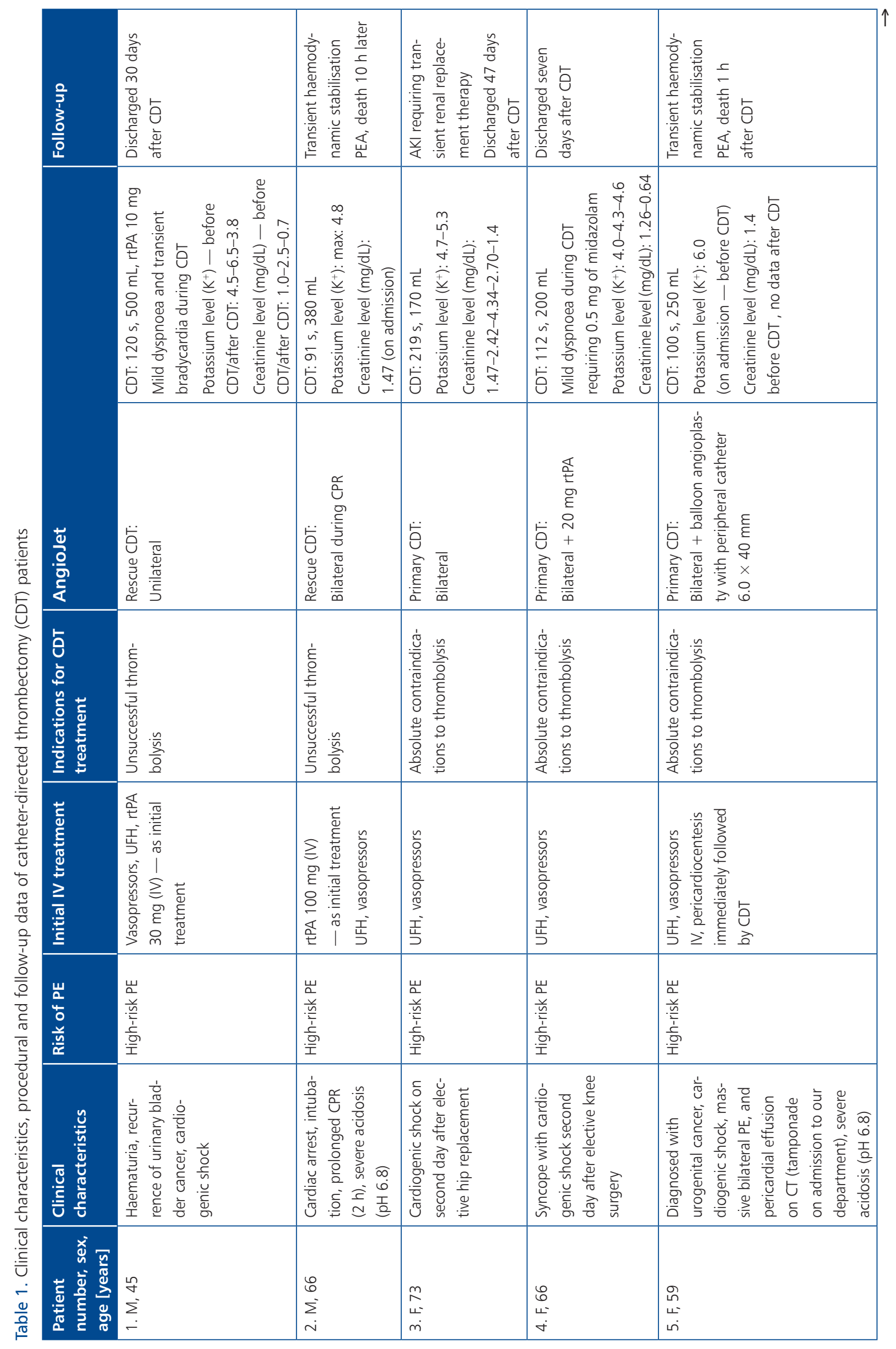




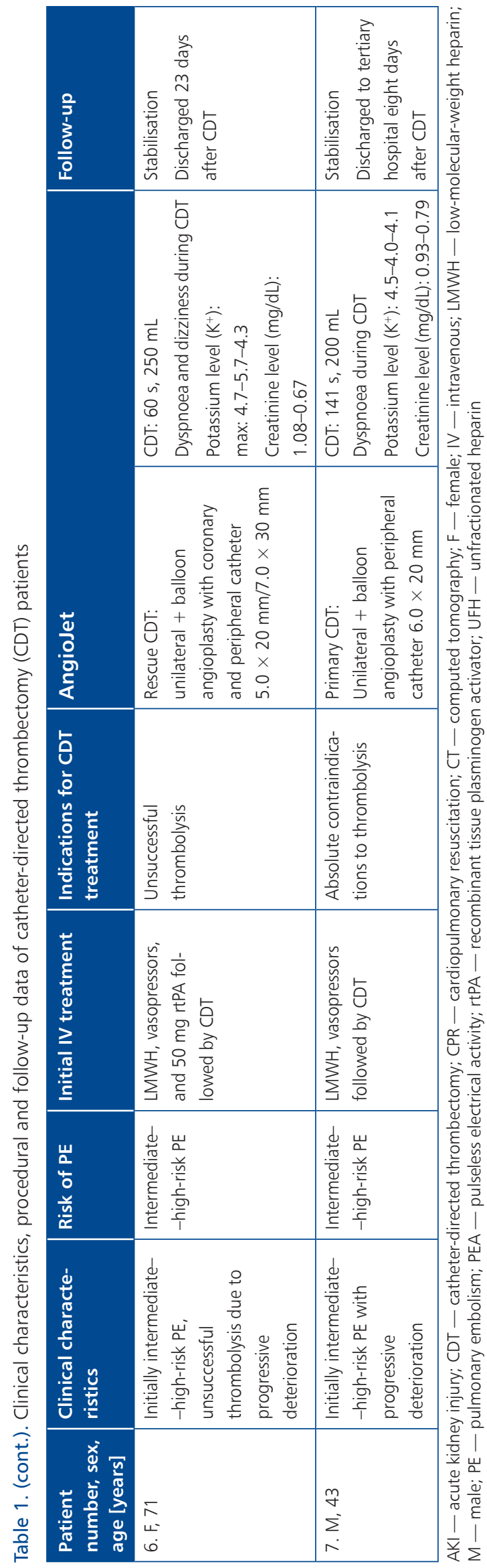

\section{RESULTS AND DISCUSSION}

Between May 2015 and March 2018, 291 patients with PE were admitted to our department, of whom 193 were diagnosed with intermediate-risk PE (120 patients classified as intermediate-high-risk and 73 as intermediate-low-risk) and 15 with high-risk PE. Thrombolysis was performed in 16 patients and CDT in seven patients (Table 1). Haemodynamic data before and after CTD are presented in Supplemental Table 2 (see journal website). An example of a CDT intervention is presented in Supplemental Figure 1 (see journal website). Despite ongoing significant improvement in the management of $\mathrm{PE}$, morality rate in the high-risk group still remains unacceptably high. Haemodynamic instability affects $5 \%$ to $10 \%$ of all PE cases and is a strong indication for urgent revascularisation [1-3]. Systemic thrombolysis is preferred, while surgical embolectomy is recommended when thrombolysis is contraindicated or has failed. Percutaneous embolectomy has become a treatment option for patients with high-risk PE [1]. There are several catheter intervention techniques, including thrombus fragmentation, suction thrombectomy, rheolytic thrombectomy, conventional CTD, and ultrasound-assisted catheter-directed low-dose thrombolysis. There is no single, generally accepted technique for CTD in high-risk patients.

We report seven haemodynamically unstable patients treated with the Angiojet system, which generates a high-pressure saline jet with a pressure gradient calculated by Bernoulli's principle, enabling the removal of thrombus fragments. The AngioJet system has received the United States Food and Drug Administration (FDA) approval and the Conformité Européenne (CE) mark for treatment of proximal deep vein thrombosis, as well as the CE mark for PE treatment. Transient release of bradykinin, adenosine, or potassium secondary to haemolysis may lead to dyspnoea, bradycardia, or even heart block. In some cases, haemoglobinuria with acute kidney injury was reported, which resulted in a FDA black-box warning for the use of AngioJet in PE [14, 15]. Importantly, technical improvements of the Angiojet system and shortening of its activation time prevented these complications [15]. Four of our subjects had contraindications to thrombolysis: recent major surgery, multiorgan injury, or cardiac tamponade, while in the remaining three patients systemic thrombolysis failed. Five of seven urgently treated patients with high-risk PE survived and were discharged in good general condition. Two patients died despite transient haemodynamic improvement. We think that irreversible cardiogenic shock and multisystem organ failure due to prolonged cardiopulmonary resuscitation and long-lasting systemic hypoperfusion caused multiorgan failure with severe life-threatening acidosis $(\mathrm{pH}<6.9)$, which led to a fatal outcome despite haemodynamic improvement. Importantly, we observed only one major complication in survivors. An elderly woman, due to contrast media nephropathy and probably haemolysis during CDT, experienced acute kidney injury requiring transient renal replacement 
therapy. However, several months earlier she had developed contrast-induced nephropathy (with an estimated glomerular filtration rate nadir of $20 \mathrm{~mL} / \mathrm{min}$ ) after an elective invasive vascular procedure. No significant hyperkalaemia was detected after CDT.

This is a case-based, observational study with a relatively small number of patients and interventions. Due to the lack of a department of cardiac surgery in our institution and a very limited immediate availability of surgical embolectomy, our local PERT with CDT capabilities does not include a cardiac surgeon.

In conclusion, we are convinced that CDT is potentially lifesaving in patients with high-risk PE and contraindications to fibrinolysis as well as those after failed fibrinolysis. There is undoubtedly a need for further studies assessing the efficacy and safety of this technique in patients with PE.

\section{Conflict of interest: none declared}

\section{References}

1. Konstantinides SV, Torbicki A, Agnelli G, et al. Task Force for the Diagnosis and Management of Acute Pulmonary Embolism of the European Society of Cardiology (ESC). 2014 ESC guidelines on the diagnosis and management of acute pulmonary embolism. Eur Heart J. 2014; 35(43): 3033-3069, doi:10.1093/eurhearti/ehu283, indexed in Pubmed: 25173341.

2. Kucher N, Rossi E, Rosa MDe, et al. Massive pulmonary embolism. Circulation. 2006; 113(4): 577-582, doi: 10.1161/circulationaha.105.592592.

3. Labyk A, Ciurzyński M, Jankowski K, et al. Acute pulmonary embolism: analysis of consecutive 353 patients hospitalised in a single centre. A 3-year experience. Kardiol Pol. 2012; 70(1): 15-22, indexed in Pubmed: 22267418.

4. Chauhan CA, Scolieri SK, Toma C. Percutaneous pulmonary embolectomy using the flowtriever retrieval/aspiration system. J Vasc Interv Radiol. 2017; 28(4): 621-623, doi: 10.1016/j. jvir.2016.09.012, indexed in Pubmed: 28343592.

5. Kuo WT, Banerjee A, Kim PS, et al. Pulmonary Embolism Response to Fragmentation, Embolectomy, and Catheter Thrombolysis (PERFECT): Initial Results From a Prospective Multicenter Registry. Chest. 2015; 148(3): 667-673, doi: 10.1378/chest.15-0119, indexed in Pubmed: 25856269.

6. Piazza G, Hohlfelder B, Jaff MR, et al. A prospective, single-arm, multicenter trial of ultrasound-facilitated, catheter-directed, low-dose fibrinolysis for acute massive and submassive pulmonary embolism: the SEATTLE II study. JACC Cardiovasc Interv. 2015; 8(10): 1382-1392, doi:10.1016/j.jcin.2015.04.020, indexed in Pubmed: 26315743.

7. Jaber WA, Fong PP, Weisz G, et al. Acute Pulmonary Embolism: With an Emphasis on an Interventional Approach. J Am Coll Cardiol. 2016; 67(8): 991-1002, doi: 10.1016/j.jacc.2015.12.024, indexed in Pubmed: 26916490.

8. Dudzinski DM, Giri J, Rosenfield K. Interventional Treatment of Pulmonary Embolism. Circ Cardiovasc Interv. 2017; 10(2): 1-10, doi:10.1161/CIRCINTERVENTIONS.116.004345, indexed in Pubmed: 28213377.

9. Stępniewski J, Kopeć G, Magoń W, et al. Ultrasound-assisted, catheter-directed, low-dose thrombolysis for the treatment of acute intermediate-high risk pulmonary embolism. Pol Arch Intern Med. 2018; 128(6): 394-395, doi: 10.20452/pamw.4272, indexed in Pubmed: 29806658.

10. Roik M, Wretowski D, Machowski M, et al. Successful treatment of intermediate-high-risk pulmonary embolism with aspiration thrombectomy: first experience in Poland. Kardiol Pol. 2018; 76(9): 1381, doi: 10.5603/KP.2018.0190, indexed in Pubmed: 30211948.

11. Witkin AS. Acute and chronic pulmonary embolism: the role of the pulmonary embolism response team. Curr Opin Cardiol. 2017; 32(6): 672-678, doi:10.1097/HCO.0000000000000455, indexed in Pubmed: 28786863.

12. Serhal M, Haddadin IS, Heresi GA, et al. Pulmonary embolism response teams. J Thromb Thrombolysis. 2017; 44(1): 19-29, doi: 10.1007/s11239-017-1498-9, indexed in Pubmed: 28401327.

13. Latacz P, Simka M, Brzegowy P, et al. Treatment of high- and intermediate-risk pulmonary embolism using the AngioJet percutaneous mechanical thrombectomy system in patients with contraindications for thrombolytic treatment - a pilot study. Wideochir Inne Tech Maloinwazyjne. 2018; 13(2): 233-242, doi: 10.5114/wiitm.2018.75848, indexed in Pubmed: 30002757.

14. Kuo WT, Gould MK, Louie JD, et al. Catheter-directed therapy for the treatment of massive pulmonary embolism: systematic review and meta-analysis of modern techniques. J Vasc Interv Radiol. 2009; 20(11): 1431-1440, doi: 10.1016/j.jvir.2009.08.002, indexed in Pubmed: 19875060.

15. Das S, Das N, Serota H, et al. A retrospective review of patients with massive and submassive pulmonary embolism treated with AngioJet rheolytic thrombectomy with decreased complications due to changes in thrombolytic use and procedural modifications. Vascular. 2018; 26(2): 163-168, doi:10.1177/1708538117722728, indexed in Pubmed: 28828935.

Cite this article as: Roik M, Wretowski D, Łabyk A, et al. Initial experience of pulmonary embolism response team with percutaneous embolectomy in intermediate-high- and high-risk acute pulmonary embolism. Kardiol Pol. 2019; 77(2): 228-231, doi: 10.5603/KP.a2018.0239. 